\begin{tabular}{|c|c|c|}
\hline \multicolumn{3}{|c|}{ DJS Vol. 38 (2017) 1-6 } \\
\hline$\frac{1969}{\int_{2016}}$ & $\begin{array}{c}\text { Delta Journal of Science } \\
\text { Available online at } \\
\text { https://djs.journals.ekb.eg/ }\end{array}$ & 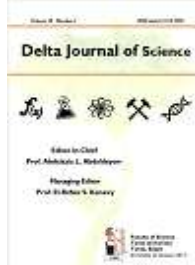 \\
\hline Research Article & & HEMATI \\
\hline
\end{tabular}

\title{
Relative convex body in Euclidean and hyperbolic spaces
}

\author{
H. K. El-sayied, M. Swealam and Doaa Mohammed El-sayed \\ Department of Mathematics, Faculty of Science, Tanta University, Tanta, Egypt
}

\begin{abstract}
This paper is to generalize the concept of convex body to the so called relative convex body in Euclidean space $E^{n}$. Some geometrical and topological properties for this kind of sets are discussed. Some properties of the central projection map(Beltrami map) introduced to discuss these concepts in the hyperbolic space $H^{n}$..
\end{abstract}

Key words: Relative convexity, relative open (closed) sets, relative convex body and relative convex surface.

\section{Introduction:}

The concept of convex bodies have an important role in differential geometry and represent a very interesting fruitful area of research.

In the last years a lot of mathematicians generalized convexity notion in Euclidean space $E^{n}$. Such as K-convexity[4], Dstarshaped sets[3], Invexity[2], and Relative convexity[5].

A new kind of generalized convex body for sets in Euclidean and hyperbolic spaces is presented, this kind is called relative convex body. Also some geometrical and topological properties for this kind are discussed. Before this discussion let us survey some definitions and results that help us in this work.

\section{Definition(1).}

A subset $\mathrm{B}$ of the Euclidean space $E^{n}$ is an open set if it consists entirely of interior points $\operatorname{in}(B)$, hence if $B=\operatorname{in}(B)$. A subset $B C E^{n}$ is a closed set if it contains its boundary $B d(B)$, hence if $B$ J $B d(B)$.[7]

Theorem(2).

(1)The Euclidean space $E^{n}$ and the empty set are open (closed) sets.
(2)The union of any number (finite number)of open (closed) sets is an open (closed) set.

(3)The intersection of a finite number (any number) of open (closed) sets is an open (closed) set.

\section{Theorem(3).}

The following statments are equivalent

(1) $B$ is a closed set, that is $B \supset B d(B)$.

(2)The limit points to $B, \operatorname{Lp}(B)$, belong to $B$, that is $B \supset \operatorname{Lp}(B)$.

(3)If the neighbourhood $N(p, \delta) \cap B \neq$ $\emptyset, \forall \delta \geq 0$, then $p \in B$.

(4)The complement of $B$ is an open set.

(5) $B$ is its own closure, $\operatorname{Cl}(B)$, that is $B=$ $C l(B)$.

Where $N(p, \delta)=x \in B: d(p, x) \leq \delta .[7]$ 
Theorem(4).

For any set $B$, the following statments are hold:

(1)The interior $\operatorname{In}(B)$ and the exterior $\operatorname{Ex}(B)$ are open sets, hence $\operatorname{In}[\operatorname{In}(B)]=\operatorname{In}(B)$.

(2)The closure $\operatorname{Cl}(B)$ is a closed set, hence $C l[C l(B)]=C l(B)$.

(3)The boundary $B d(B)$ is a closed, hence $B d[B d(B)] C B d(B)$.

(4)The derived set $\operatorname{Lp}(B)$ is a closed set, hence $\operatorname{Lp}[\operatorname{Lp}(B)] C \operatorname{Lp}(B)$.[7]

\section{Definition(5).}

A subset $S C E^{n}$ is a convex set if for each pair of points $x, y \in S$ it is true that the closed line segment $[x y]$ joining $x$ and $y$ lies wholly in $S .[1,8]$

\section{Definition(6).}

Let $B$ be a subset of the Euclidean space $E^{n}$ and $A$ be a subset of $B$. The set $A$ is a relatively convex set with respect to $B$ if for each pair of points $p, q \in A$ the closed segment $[p q]$ joining $p$ and $q$ lies wholly in B.[5]

In the following some results are introduced as given in [5].

(1)If $A_{1}$ and $A_{2}$ are relatively convex with respect to $B$, then $A_{1} \cap A_{2}$ relatively convex with respect to $B$. On the other hand for $A_{1} \cup$ $A_{2}$ the above result is no longer valid.

(2)Every subset $A C E^{n}$ is relatively convex with respect to any of its supersets.

(3)Every subset $A$ is relatively convex with respect to its convex hull. Moreover, each subset is relatively convex with respect to any convex superset.

(4)If $A$ is a relatively convex set with respect to $B$, then every subset of $A$ is relatively convex with respect to $B$.

(5)Let $A C E^{n}$ be a subset. If every subset $B C A$ is relatively convex with respect to $A$, then $A$ is convex.
4-Relative closed and relative open sets

In the following section, we shall introduce some definitions and results on relative closed and relative open sets.

\section{Definition(7).}

Let $B$ be a subset of the Euclidean space $E^{n}$ and $A$ be a subset of $B$. The set $A$ is said to be a relatively closed with respect to $B$ if every limit point of $A$ belongs to $B$.

We denote to the set of all limit points of the set $A$ by $A^{\prime}$. Which is called the derived set.[7]

\section{Proposition(8).}

The empty set is relative closed set with respect to any set $A$.

\section{Proof}

Let the empty set $\varnothing$ be not relative closed with respect to $A$. Then, there exists a limit point of $\emptyset$, say $x$, such that $x$ dose not belong to $A$. Since the empty set has no limit points, Then this is a contradiction. Therefore, the empty set is relative closed with respect to any set $A$.

\section{Corollary(9).}

The Euclidean space is relative closed with respect to itself.

\section{Proposition(10).}

If $A_{1}$ and $A_{2}$ are relative closed with respect to $\mathrm{B}$, then $A_{1} \cap A_{2}$ and $A_{1} \cup A_{2}$ are also relative closed with respect to $B$.

\section{Proof}

Firstly, since $A_{1}$ and $A_{2}$ are relative closed with respect to $B$, then $A_{1}, A_{2} C B$ and $A_{1}^{\prime}, A_{2}^{\prime} C B$, Thus $\left(A_{1} \cap A_{2}\right) C B$. Let $x$ be a limit point of $A_{1} \cap A_{2}$ then, $x \in\left(A_{1} \cap\right.$ $\left.A_{2}\right)^{\prime} \underline{\mathrm{C}}\left(A_{1}^{\prime} \cap A_{2}^{\prime}\right)$, this implies that $x \in$ $A_{1}^{\prime} \wedge x \in A_{2}^{\prime}$, then $x \in B$. Hence $A_{1} \cap A_{2}$ is relative closed with respect to $B$. Secondly, simillarly we have $\left(A_{1} \cup A_{2}\right) \subset B$ and $A_{1}^{\prime} \cup$ $A_{2}^{\prime} \mathrm{C} B$, if $x$ is a limit point of $\left(A_{1} \cup A_{2}\right)$, then $x \in\left(A_{1} \cup A_{2}\right)^{\prime}=\left(A_{1}^{\prime} \cup A_{2}^{\prime}\right)$. Therefore $x \in$ $A_{1}^{\prime}$ or $x \in A_{2}^{\prime}$. Therefore $x \in A_{1}^{\prime}$ or $x \in A_{2}^{\prime}$. Therefore, $x \in B$. Hence, $A_{1} \cup A_{2}$ is relative closed with respect to $B$. 


\section{Proposition(11).}

The set $A$ is relative closed set with respect to itself if and only if $A$ is closed.

\section{Proof}

(1)If $A$ is closed, then $A$ contains all limit points of $A$. Hence $A$ is relative closed with respect to $A$.

(2)If $A$ is relative closed with respect to itself, then $A$ contains all limit points of $A$. Thus, $A$ is closed.

\section{Proposition(12).}

If $A$ is relative closed with respect to $B$, then the closure of $A$ is relative closed with respect to the closure of $B$.

\section{Proof}

Since $A$ is relative closed with respect to $B$, then we have $A C B$ and $A^{\prime} C B$. This implies that $A C \operatorname{Cl}(A) \operatorname{Cl}(B)$. Since $\operatorname{Cl}(A)$ is closed, then $(\operatorname{Cl}(A))^{\prime} \mathrm{C} C l(A) \mathrm{C} C l(B)$, hence $\operatorname{Cl}(A)$ is relatively closed with respect $C l(B)$.

\section{Remark(13).}

(1) Every closed set $A$ is relative closed with respect to any of its supersets.

(2)Every subset is relative closed with respect to any closed superset.

\section{Corollary(14).}

Every set $A$ is relative closed with respect to $\operatorname{Cl}(A)$.

\section{Proposition(15).}

If $A$ is a relatively closed with respect to $B$, then every subset of $A$ is relatively closed with respect to $B$.

\section{Proof}

Let $x$ be any limit point of $C C A C B$, then for all open set $G$ containing $x$, we have ( $G-$ $x) \cap C \neq \varnothing$ implies $(G-x) \cap A \neq \emptyset$. This means that $x$ is a limit point for $A$. Since $A$ is relative closed with respect to $B$. Hence, $x$ belongs to $B$. Therefore, $C$ is relative closed with respect to $B$.

\section{Corollary(16).}

If $A$ is relative closed set with respect to $B$, then the interior of $A$ is relative closed with respect to $B$.

\section{Lemma(17).}

If $A$ is relative closed set with respect to $B$ and $C$, then $A$ is relative closed with respect to both $B \cap C$ and $B \cup C$.

\section{Proof}

Since $A$ is relative closed with respect to $B$ and $C$, then we have

$$
\begin{aligned}
& A \subset B, A^{\prime} C B(1) \text {. And } \\
& \qquad A \subset C, A^{\prime} \subset C(2) .
\end{aligned}
$$

From (1) and (2), we have $A C(B \cap C)$ and $A^{\prime} C(B \cap C)$. Hence, $A$ is relative closed with respect to $B \cap C$. Simillarly, it is easy to see that $A$ is relative closed with respect to $B \cup C$.

\section{Theorem(18).}

If $A$ and $B$ are two relative closed sets with respect to $C$, then $A \cap B$ and $A \cup B$ are also relative closed sets with respect to $C$.

\section{Proof}

Since $A$ and $B$ are relative closed with respect to $C$. Then

$A \subset C, A^{\prime} C C(1)$. And

$B C C, B^{\prime} C C(2)$.

From (1) and (2), we have $(A \cap B) C C$ and $\left(A^{\prime} \cap B^{\prime}\right) C C$. Therefore $(A \cap B)^{\prime} C C$. Hence $(A \cap B)$ is relative closed set with respect to $C$.

Simillarly, it is easy to see that $(A \cup B)$ is relative closed with respect to $C$.

\section{Proposition(19).}

If $A$ is relative closed set with respect to both $B$ and $C$ such that $B \not C$. Then, there exists a proper subset $W C B$ such that $A$ is relative closed set with respect to $W$.

\section{Proof}

(1)If $C$ C $B$, we put $W=C$. Hence, $A$ is relative closed with respect to $W$.

(2)If $C \not \subset B$ and $B \not C C$ (given), then we have $(B \cap C) C B$. Let $W=(B \cap C), W$ is a proper subset of $B$. Since $A$ is relative closed with respect to both $B$ and $C$. From the above lemma, we see that $A$ is relative closed with respect to $W$. 
Proposition(20).

Let $A$ be a subset of the Euclidean space $E^{n}$. If every subset of $A$ is relative closed with respect to $A$, then $A$ is closed.

\section{Proof}

If $B C A$ is a relative closed with respect to $A$, then $A$ contains all the limit points of the subset $B$. This is also true when we take $B=$ $A$,i.e. $A$ contains all the limit points of $A$. Therefore, $A$ is closed.

\section{Definition(21).}

Let $B C E^{n}$ and $A C B$. The set $A$ is called relative open set with respect to $B$ if for all $x \in A$, there exists an open set $G$ containing $x$ such that $x \in G C B$, i.e; $G$ is entirely in $B$.

\section{Remark(22).}

(1)The empty set is relative open with respect to any set.

(2)The Euclidean space $E^{n}$ is relative open with respect to $E^{n}$.

(3)The interior of a set $A$ is relative open with respect to $A$.

(4)Let $A C E^{n}$ be a subset. If every subset $B C A$ is relative open with respect to $A$. Then $A$ is open.

\section{Proposition(23).}

The set $A$ is relative open with respect to $A$ if and only if $A$ is open.

\section{Proof}

(1)If $A$ is open, then for all $x \in A$, there exists an open set $G$ containing $x$ such that $x \in G C A$. Thus $A$ is relative open with respect to $A$.

(2)If $A$ is relative open with respect to itself, then for all $x \in A$, there exists an open $\operatorname{set} G$ containing $x$, such that $x \in G C A$. This means that $A$ is open.

\section{Theorem(24).}

If the two sets $A_{1}$ and $A_{2}$ are relative open with respect to $B$, then $A_{1} \cap A_{2}$ and $A_{1} \cup A_{2}$ are relative open with respect to $B$.

\section{Proof}

(1)Since $A_{1}$ and $A_{2}$ are relative open with respect to $B$, then we have $A_{1} \subset B$ and $A_{2} \subset B$, thus $\left(A_{1} \cap A_{2}\right) \subset B$. If $x \in\left(A_{1} \cap A_{2}\right)$, then $x \in A_{1} \wedge x \in A_{2}$. Again since $A_{1}$ and $A_{2}$ are relative open with respect to $B$, then there exist the open sets $G_{1}$ and $G_{2}$ such that $x \in$ $G_{1} C B$ and $x \in G_{2} C B$. Thus $x \in$ $\left(G_{1} \cap G_{2}\right) \subset B$. Therefore $A_{1} \cap A_{2}$ is relative open with respect to $B$.

(2)Since $A_{1} C B$ and $A_{2} \subset B$, then $\left(A_{1} \cup\right.$ $\left.A_{2}\right) \subset B$. Let $x \in\left(A_{1} \cup A_{2}\right)$, then $x \in A_{1}$ or $x \in A_{2}$. Since $A_{1}$ and $A_{2}$ are relative open with respect to $B$, then we discuss the following cases:

(a)If $x \in A_{1}$, then there exists an open set $G_{1}$ containing $x$ such that $x \in G_{1} \subset B$, then $A_{1} \cup$ $A_{2}$ is relative open with respect to $B$.

(b)If $x \in A_{2}$, then there exists an open set $G_{2}$ containing $x$ such that $x \in G_{2} \subset B$, then $A_{1} \cup$ $A_{2}$ is relative open with respect to $B$.

(3)If $\left(A_{1} \cap A_{2}\right) \neq \varnothing$. In this case, we see that $\emptyset$ is relative open with respect to $B$.

\section{Proposition(25).}

If $A$ and $B$ are two relative open sets with respect to $C$, then $(A \cap B)$ and $(A \cup B)$ are relative open with respect to $C$.

\section{Proof}

For all $x \in(A \cap B)$, we have $x \in A \wedge x \in B$. Since $A$ and $B$ are relative open with respect to $C$, then there exist the open sets $G_{1}$ and $G_{2}$ such that $x \in G_{1} C C$ and $x \in G_{2} C C$. Hence, $x \in\left(G_{1} \cap G_{2}\right) C C$. Therefore, $A \cap B$ is relative open with respect to $C$. Simillary, it is easy to see that $A \cup B$ is relative open with respect to $C$.

\section{5-Relative convex body}

In this section, we define relative convex body and study some geometrical properties for this concept.

\section{Definition(26).}

Let $B$ subset of $E^{n}$, and $A C B$. If the subset $A$ is bounded and relative closed with respect to $B$, then $A$ is called relative compact with respect to $B$.

\section{Remark(27).}

(1)If $A$ is relative compact set with respect to $B$, then every subset of $A$ is relative compact set with respect to $B$. 
(2)If $A$ is relative compact set with respect to $B$ and $C$, then $A$ is relative compact set with respect to both $B \cap C$ and $B \cup C$.

\section{Definition(28).}

Let $A C B, A$ is called relative convex body with respect to $B$ if the following conditions are satisfied:

(1) $A$ is relative compact with respect to $B$.

(2) $A$ is relative convex with respect to $B$.

(3) $A$ has non-empty interior.

\section{Definition(29).}

The relative convex surface is the boundary of the relative convex body.

It is easy to see that

(1)if the two sets $A_{1}$ and $A_{2}$ are relative convex bodies with respect to $B$. Then, we have

i) $\left(A_{1} \cap A_{2}\right) \neq \varnothing$ is relative convex body with respect to $B$. (

ii) $A_{1} \cup A_{2}$ is not relative convex body in general.(

(2)Every non-empty subset $A C E^{n}$ is relatively convex body with respect to $E^{n}$.

(3)Every convex body is relatively convex body with respect to any of its supersets.

(4)If $A$ is a relatively convex body with respect to $B$, then every non-empty bounded subset of $A$ is relatively convex body with respect to $B$.

Proposition(30).

If $A$ is a relative convex body with respect to both $B$ and $C$, such that $B$ is not a subset of $C$. Then there exists a proper subset $W C B$ such that $A$ is relative convex body with respect to $W$.

\section{Proof}

(1)Assume that $C$ C $B$ and put $W=C$. Hence $A$ is relative convex body with respect to $W$.

(2)Assume that $C \not B$ and( $B \not C$, given), let $W=(B \cap C)$, clearly $W$ is a proper subset of $B$. Since $A$ is relative convex body with respect to both $B$ and $C$. Therefore, we have $A$ is relative convex body with respect to $W$.

\section{6-Relative convex in hyperbolic space}

Now we devote our study to the concept of relative convex in hyperbolic space $H^{n}$. The most convenient model of the $n$-dimensional hyperbolic space for the present work is the spherical one $H^{n}$ which might be defined as follows [8],[5]:for

$$
\left.H^{n}=\left(x^{1}, x^{2}, \ldots \ldots x^{n}\right) \in V^{n+1}:-\left(-\left(x^{1}\right)^{2}+\sum_{i=2}^{n+1} x^{i}\right)^{2}\right)=1 \quad, x^{1} \succ 0
$$

and also in the metric, where $V^{n+1}$ denotes the Minkowski space $\left(R^{n+1},<,>\right)$ with the pseudo-Riemannian metric $<,>=$ $-d x^{1} \odot d x^{1}+\sum d x^{i} \odot d x^{i}$. The metric when restricted to $H^{n}$ yields a Riemannian metric with constant sectional curvature $K=-1$ [5]. As $H^{n}$ is a complete simply connected Riemannian manifold with negative sectional curvature, then each pair of points $p, q \in H^{n}$ are joined with a unique geodesic segment [8]. Therefore, $H^{n}$ is starshaped. The Beltremi(or central projection)map $\beta: H^{n} \rightarrow$ $E^{n}$ is defined to be the map which takes $x \in$ $H^{n}$ to the intersection of the Euclidean space $x^{1}=1$ with the straight line through $x$ and the origin 0 of $V^{n+1}$. The map $\beta$ takes the whole of $H^{n}$ diffeomorphically to the open ball $B(p, l)$ of radius 1 and center at $p=$ $(1,0,0, \ldots, 0)$. Furthermore, the map $\beta$ is a geodesic map and so K-totally geodesic submanifolds of $H^{n}$ are mapped under $\beta$ onto k-planes in $E^{n}$. We can also show that closed, open, compact, bounded and starshaped subsets of $H^{n}$ are mapped under $\beta$ to subsets of $B(p, l)$ of the same type. It worth mentioning that the inverse of map $\beta$ has the same properties of $\beta$.[6]

\section{Lemma(31).}

The central projection map preserves limit points of sets.

\section{Proof}

Obvious.

\section{Proposition(32).}

The central projection map preserves relative closed property of set.

\section{Proof}

Let $B$ be a subset of $H^{n}$ and $A$ is a relative closed set with respect to $B$. Then, for any limit point of $A$, say $x$, belongs to $B$. If we apply the central projection map $\beta$, then we have $\beta(x)$ is a limit point of $\beta(A)$ and $\beta(A) \subset \beta(B)$. Since $x \in B$, then $\beta(x) \in$ $\beta(B)$. Therefore, $\beta(x)$ is a limit point of 
$\beta(A) \subset \beta(B)$ and $\beta(x) \in \beta(B)$. Hence, $\beta(A)$ is relative closed set with respect to $\beta(B)$.

\section{Proposition(33).}

The central projection map preserves on relative open sets.

\section{Proof}

Let $B$ be a subset in $H^{n}$ such that is relative open set with respect to $B$. Then, for any $x \in$ $B$ and there exists an open set $G$ containing $x$ such that $x \in G C B$. If we apply $\beta$ then $\beta(x) \in G^{\prime} C \beta(B)$. Therefore the centeral projection map preserves on relative open sets, where $\beta(G)=G^{\prime}$ is open set.

\section{Proposition(34).}

The central projection preserves on relative convexity.

\section{Proof}

Let $B$ be a closed connected set in $H^{n}$ and $A$ be a subset of $B$. Assume that $x, y$ in $A$ and the closed geodesic segment ,say $\alpha x y$, which is determined by $x$ and $y$ is in $B$. Then if we apply $\beta$ and assume that the closed segment $[\beta(x) \beta(y)]$ is not in $\beta(B)$,

this means that there exists at least one point belongs to $[\beta(x) \beta(y)]$ but not belongs to $\beta(B)$, which is a contradiction with the fact that $\beta$ preserves on the interior, exterior and boundary points of $B$. Hence $\beta(x), \beta(y)$ are in $\beta(A)$ and $[\beta(x) \beta(y)]$ is in $\beta(B)$. This implies that $\beta(A)$ is relative convex with respect to $\beta(B)$.

\section{Corollary(35).}

the central projection map preserves on the concept of relative convex body.

\section{References}

[1]F.A.Valentine: Convex sets, Mc Graw Hill, New York, 1964.

[2]H.K.El-Sayied: Invex bodies and focal points, Comp. Sci. Vol.9, No.1, (1996), 25-28.

[3]H.K.El-Sayied: On D-Starshaped sets, Far east jor. of math. Sci. Vol.28, Issue 2, (2008), 469-481.

[4]M.Beltagy and I.Saker: K-Convexity in Euclidean 3-space, Comm.Fac.Sci. University Ankara, 44(1995), 49-58.
[5]M.Beltagy and H.K.El-Sayied: Relative Convexity in Euclidean space, TENSOR, N.S.Vol.69(2008).

[6]M.Swealam and S.Moheb, on the closed curves in Non-Euclidean space forms, Ain Shams Science Bull Egypt,Vol.29(A.)(1991):61-73.

[7]P.J.Kelly and M.L.Weiss: Geometry and Convexity, John Wiley, Sons, New york, (1979).

[8]S.R.Lay:Convexsets and their applications, John Wiely and Sons, New York, (1982). 